\title{
A comparative investigation of the pavement layer dielectrics by FDTD modelling and reflection amplitude GPR data
}

\author{
F. Tosti \& A.M. Alani \\ School of Computing and Engineering, University of West London, London, United Kingdom
}

\author{
A. Benedetto, L. Bianchini Ciampoli \& M.G. Brancadoro \\ Department of Engineering, Roma Tre University, Rome, Italy
}

L. Pajewski

Department of Information Engineering, Electronics and Telecommunications, Sapienza University of Rome, Italy

ABSTRACT: The present work focuses on the application of the ground-penetrating radar (GPR) technique on a flexible pavement structure for the assessment of the layer dielectrics. Two air-coupled GPR systems, with antennas operating at $1 \mathrm{GHz}$ and $2 \mathrm{GHz}$ central frequencies have been used for testing and simulation purposes. The effectiveness of the combination of i) the Finite-Difference Time-Domain (FDTD) technique for the simulation of the GPR signal, and ii) the GPR reflection amplitude technique, for the estimation of the dielectrics of the pavement layers, has been analyzed. Three steps of processing are proposed and the results are compared each to one another. In the first stage, the signal has been simulated using design project data for the crosssection investigated and dielectric permittivity values for the (design) construction materials, derived from the literature. In the second stage, the dielectrics have been computed by the signal collected within a real-life flexible pavement. Both the two-way travel time and the reflection amplitude techniques were performed. The third step was focused on analyzing the accuracy of the reflection amplitude method combined with the optimized simulation of the GPR signal. The results demonstrate potential on the use of the proposed approach with respect to the application of the reflection amplitude technique to the real-life GPR signal.

\section{INTRODUCTION}

The effective measurement of layer thicknesses is crucial for quality control purposes, rehabilitation and maintenance actions of road infrastructures. Indeed, the pavement layer thicknesses are basic information within the implementation of mechanistic models for the assessment of the pavement performance as well as of the structural tests (Scullion \& Saarenketo 2000).

Ground-penetrating radar (GPR) has been extensively used in highway engineering over the last four decades for the assessment of the layer thicknesses. The increasing sophistication of the operational practices along with the enhancements of the technology have made this technique as established (ASTM 2015) and prevailing over other destructive (e.g., coring) or low productive non-destructive testing (NDT) techniques (e.g., the falling weight deflectometer (FWD)) (Al-Qadi \& Lahouar 2005).

GPR is a remote sensing electromagnetic (EM) device that emits a short pulse of EM waves toward a surface, e.g., a pavement surface. Overall, groundcoupled and air-coupled antenna systems are used for pavement evaluation purposes.
The ground-coupled systems are equipped with antennas resting on the pavement surface. Notwithstanding the limited speed of inspection, such systems allow for higher penetration depths.

The air-coupled systems operate at driving speeds with antennas suspended above the pavement surface. Despite their higher productivity, the penetration depth is more limited than in the ground-coupled systems. Ideally, the air-coupled systems should be used to get information about the surface layers of the pavement, whereas the ground-coupled systems should be utilized for retrieving information about the load-bearing layers, up to approximately $3 \mathrm{~m}$ depth, on the average (Loizos \& Plati 2007a). In real-world applications, the air-coupled antenna systems are the most widely used, since they can avoid for the total or partial closure of the carriageway at driving speeds of investigation. To that effect, the estimation of the layer thicknesses are often performed using $1 \mathrm{GHz}$ (Willet et al. 2006) and alternatively $2 \mathrm{GHz}$ central frequency antennas (Loizos \& Plati 2007b). This depends on, among the other, the layer to measure, the expected layer depth from the pavement surface and the dielectric contrast between the materials of two adjacent layers.

Over the last decades many studies have focused on the estimation of the layer thicknesses (Maser 
1996, Spagnolini \& Rampa 1999, Fauchard 2001, AlQadi \& Lahouar 2004, Bastard et al. 2007, Tosti et al. 2014, Zhao et al. 2015). Among the most established approaches for the assessment of the dielectric permittivity of the layers, it is worth reminding the calibration of the wave velocity of propagation into the medium using information from reference cores (Livneh \& Siddiqi 1992) and the use of the amplitude values of the signal reflections at the interfaces of the layers (Maser \& Scullion 1991).

More recently, the use of the finite-difference time-domain (FDTD) technique (Yee 1966) for the numerical simulation of the GPR signal (Taflove 1995) has revealed to be effective in reproducing and controlling the physical and performance properties of the pavement in very complex scenarios (Giannopoulos \& Diamanti 2004, Benedetto et al. 2014, Shangguan and Al-Qadi 2015).

\section{OBJECTIVES AND METHODOLOGY}

The present work investigates on the application of the GPR technique on a flexible pavement structure for the assessment of the dielectric properties of the layers.

Two air-coupled GPR systems, with antennas operating at $1 \mathrm{GHz}$ and $2 \mathrm{GHz}$ central frequencies have been utilized. To this purpose, the effectiveness of combining i) the FDTD technique for the simulation of the GPR signal, and ii) the GPR reflection amplitude technique, for the estimation of the dielectrics of the pavement layers, has been analyzed.

In the first stage, the signal has been simulated using design project data for the cross-section investigated and the dielectrics of the (design) construction materials derived from the literature (Saarenketo 2006).

In the second stage, the dielectrics have been calculated by following two main approaches and using the signal collected in a real-life flexible pavement. In one approach, the actual thicknesses of the layers were initially measured by coring, and the dielectric permittivity of each pavement layer was then calculated after a calibration of the value of the wave propagation velocity in the medium (i.e., two-way travel time assessment of the permittivity). In the other approach, the dielectric values of the layers were assessed using the amplitudes of the reflected pulses (i.e., non-destructively).

The third step was aimed at checking the accuracy of the reflection amplitude method combined with the optimized simulation of the GPR signal. Such a simulation was run by using both geometric and dielectric features extracted from the previous steps.

The major findings of the investigation and the relevant discussion are presented in the following sections.

\section{GPR OPERATION PRINCIPLES AND THEORETICAL BACKGROUND}

\subsection{Working principles}

The GPR technique is based on the transmission of a short EM pulse sent into the pavement. The pulse travelling through the layers is reflected by surfaces or objects, which have discontinuities in the electrical properties (Figure 1).

These can be due by differing materials and/or water content and density conditions. When a contrast in the dielectrics of the pavement materials is encountered, the reflected pulses of the signal are recorded. Overall, a GPR system is composed of one antenna unit, either in monostatic (i.e., single antenna transmitting and receving) or bistatic (i.e., two separate antennas, one for the transmission and one for the reception) configuration. The control unit is the interface controlling the operation of the GPR and sending the collected data to the display unit and the data storage.

\subsection{Two-way travel time technique for the permittivity estimation}

The overall dielectric value (i.e., the relative dielectric permittivity) of a pavement layer in standard onsite conditions is given by the combination of the volumetric ratio of the material itself (asphalt and aggregates if we consider the asphalt concrete (AC) layer; soil aggregates if we consider the base layer), air and water content.

These are all weighted on the individual values of permittivity (Dobson et al. 1985, Roth et al. 1990). Correlations of the dielectric values of the AC pavement materials with the main physical (i.e., moisture and air voids), mechanical (i.e., density) and manufacturing (i.e., asphalt content) conditions have been inves

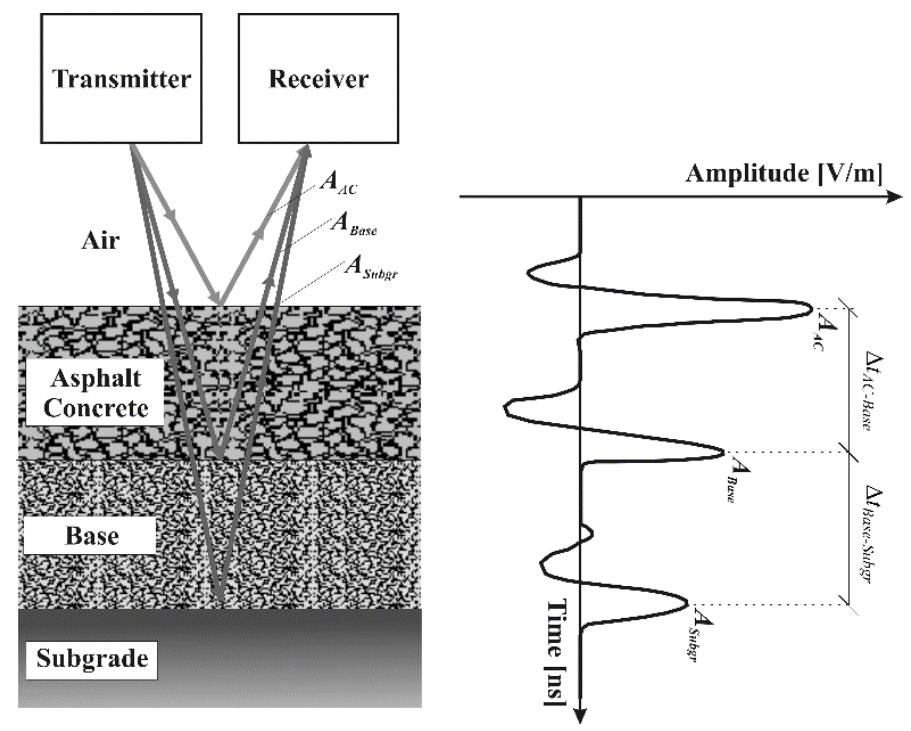

Figure 1. The GPR working principles in flexible pavements and the corresponding typical trace collected using an air-coupled antenna. 
tigated in the literature (Al-Qadi et al. 2004). Electromagnetically speaking, the velocity of propagation of an EM wave $v$ through a non-metallic material is given by:

$$
v=\frac{c}{\sqrt{\varepsilon}}
$$

where $c=$ the wave propagation velocity in the free space; and $\varepsilon=$ the relative dielectric permittivity of the medium passed through. From a kinematic point of view, once identified the relevant pulse reflections at the layer interfaces (e.g., $A_{A C}$ and $A_{\text {Base }}$ for the AC layer in Figure 1), the travel time $\Delta t$ between the pulses can be calculated. If considering the two-way path of the signal bouncing back toward the receiving antenna at the (bottom) reflection interface of the layer, the value of the wave propagation velocity $v$ can be described by:

$$
v=\frac{2 \times h}{\Delta t}
$$

where $h=$ the thickness of the pavement layer. In this paper, $v$ was computed using the thickness $h$ of each layer measured by coring. Such velocity $v$ can be subsequently worked out in Equation (1) to assess $\varepsilon$.

\subsection{Reflection amplitude technique for the permittivity estimation}

This technique relies on the proportionality between the intensity of the recorded reflections and the strength in the contrast between the dielectric properties of the pavement layers.

The amplitude peaks $A_{A C}, A_{\text {Base }}$ and $A_{\text {Subgr }}$ in Figure 1 are the reflections from the surface, base and subgrade, respectively. Such values are used for the calculation of the properties of the layers on the basis of a recursive procedure (Al-Qadi \& Lahouar 2005, Loizos \& Plati 2007b). This relies on a number of assumptions, i.e., plane wave propagation, one dimensional target geometry, and lossless layer materials illuminated by the radiated beam.

In practical terms, this technique requires the use of a large metal sheet placed on the surface of the pavement to provide a reference reflected amplitude $A_{m}$. Thereby, this reference information is utilized to determine recursively the reflection coefficients of the layer interfaces, which are used in turn to assess the dielectrics of the layers.

The dielectric values of the asphalt concrete and the base layers can be computed using the following Equations (3) and (4), respectively:

$$
\begin{gathered}
\varepsilon_{A C}=\left[\frac{1+\left(\frac{A_{A C}}{A m}\right)}{1-\left(\frac{A_{A C}}{A m}\right)}\right]^{2} \\
\varepsilon_{\text {AC Base }}=\varepsilon_{A C}\left[\frac{1-\left(\frac{A_{A C}}{A m}\right)^{2}+\left(\frac{A_{\text {Base }}}{A m}\right)}{1+\left(\frac{A_{A C}}{A m}\right)^{2}-\left(\frac{A_{\text {Base }}}{A m}\right)}\right]^{2}
\end{gathered}
$$

The dielectric value $\varepsilon_{n}$ for the third layer onwards ( $n^{\text {th }}$ layer) can be evaluated as follows:

$$
\varepsilon_{n}=\varepsilon_{n-1}\left[\frac{1-\left(\frac{A_{A C}}{A m}\right)^{2}+\sum_{i=1}^{n-2} \gamma_{i} \frac{A_{i+1}}{A m}+\frac{A_{n}}{A m}}{1-\left(\frac{A_{A C}}{A m}\right)^{2}+\sum_{i=1}^{n-2} \gamma_{i} \frac{\mathrm{A}_{i+1}}{A m}-\frac{A_{n}}{A m}}\right]^{2} \text { if } n>2
$$

where $n=$ number of the pavement layers; $A_{n}=$ the amplitude reflected by the $n^{\text {th }}$ layer interface; $\varepsilon_{n}=$ the relative dielectric permittivity of the $n^{\text {th }}$ layer; $\gamma_{i}$ is the reflection coefficient at the $i^{\text {th }}$ layer interface.

The main drawbacks of this technique are related to the main assumptions of homogeneity of the layers as well as to the lossless conditions of the manufacturing materials. This implies that the increase of the EM wave attenuation in depth through the material is not accounted for by Equations (3) to (5). Thereby, the accuracy of the dielectrics from the deeper layers may decrease.

\subsection{FDTD modelling of the GPR signal}

The effective use of the numerical simulation of the GPR signal relies on the correct solution of the Maxwell's equations, subject to the geometry of the reproduced scenario as well as on the physical properties of the materials investigated.

Within this context, the FDTD technique (Yee 1966, Kunz \& Luebbers 1993, Taflove 1995) has proved to be one of the most versatile approaches from the set of the various numerical methods available. It is based on the discretization of both the space and the time continua. Thereby, objects with continuous boundaries are represented using a staircase approximation.

In this paper, the EM simulation was run by using the FDTD technique implemented in the gprMax simulator (Giannopoulos 2005). The physical structure of the GPR antenna was not modelled. A line of current represented the source, due to the assumption of the problem invariance in one direction. To this effect, a lowering of the computational time for the data processing was reached.

\section{EXPERIMENTAL FRAMEWORK}

\subsection{Tools and equipment}

The survey was carried out using two air-coupled pulsed radar systems manufactured by IDS Georadar. Antenna systems with central frequencies of $1 \mathrm{GHz}$ and $2 \mathrm{GHz}$ were used and located at $0.40 \mathrm{~m}$ distance from the pavement surface at the rear of the van (Figure 2). Time windows of $25 \mathrm{~ns}$ and $15 \mathrm{~ns}$ were set for, respectively, the $1 \mathrm{GHz}$ and the $2 \mathrm{GHz}$ systems. After a warm up time of approximately 20 minutes, the signal reflected from the metal plate was collected and processed according to relevant ASTM standards (ASTM 2008, ASTM 2010). 


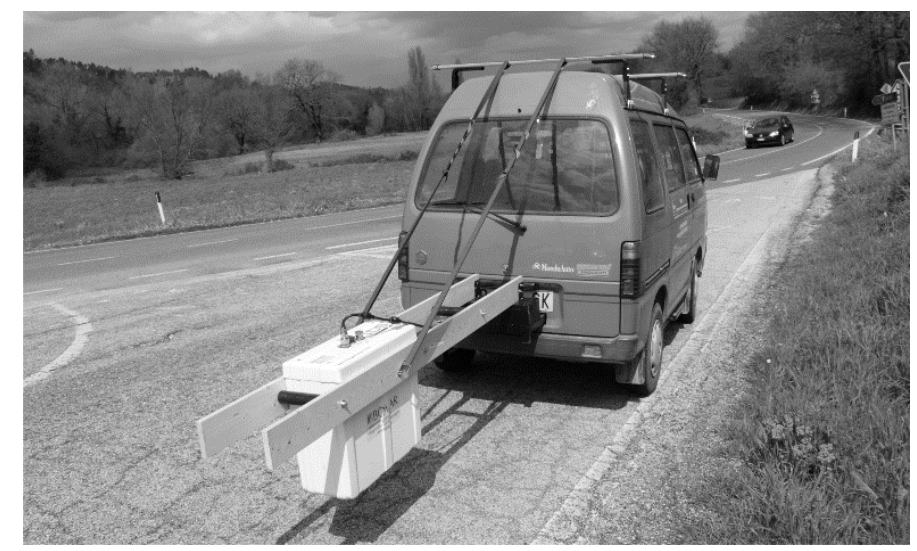

Figure 2. The GPR survey van.

\subsection{Design and coring data}

The GPR survey was conducted on an averagely trafficked two-lane highway, where recent rehabilitation works were performed. Thereby, a smooth surface was observed.

A drilled core was extracted at a position along the scanning line of the antennas. The core thicknesses were therefore compared with the design thicknesses, as given by the local Authority for the road administration (Table 1).

Table 1. Pavement design and coring data.

\begin{tabular}{llllll}
\hline \multirow{2}{*}{ Layer } & \multirow{2}{*}{ Material } & \multicolumn{3}{l}{ Thickness } \\
\cline { 3 - 6 } & & $\mathrm{c}$ Design & \multicolumn{3}{l}{ Core } \\
\hline AC & $\begin{array}{l}\text { Asphalt \& fine } \\
\text { aggregates }\end{array}$ & 6 & 2.36 & 6 & 2.36 \\
\hline AC Base & $\begin{array}{l}\text { Asphalt \& } \\
\text { coarse aggre- } \\
\text { gates }\end{array}$ & 8 & 3.15 & 5 & 1.97 \\
\hline $\begin{array}{l}\text { Granular } \\
\text { Base }\end{array}$ & $\begin{array}{l}\text { Silica aggre- } \\
\text { gates }\end{array}$ & 20 & 7.87 & 15 & 5.91 \\
\hline
\end{tabular}

\section{RESULTS AND DISCUSSION}

\subsection{Synthetic dielectrics - design data-}

The main purpose of this step is to calculate the permittivity values of the pavement layers by the simulated amplitudes of the reflected pulses (i.e., non-destructively) running the FDTD simulation of the GPR signal.

To this effect, the simulation was run in the gprMax simulator using the $1 \mathrm{GHz}$ central frequency of investigation and the design data available, as listed in Table 1 (i.e., thickness and dielectrics of the construction materials for each layer).

As regards the dielectric properties assigned to each layer, the values of dielectric permittivity $\varepsilon$ and conductivity $\sigma$ suggested by Saarenketo (2006) were used. Thereby, the Equations (3) - (5) were utilized for the calculation of the dielectrics of the AC, AC base and granular base layers (Table 2).
Table 2. Data input for the FDTD simulation and obtained synthetic permittivity values.

\begin{tabular}{|c|c|c|c|c|c|}
\hline \multirow{2}{*}{$\begin{array}{l}\text { Pro- } \\
\text { cessing } \\
\text { step I } \\
(1 \mathrm{GHz})\end{array}$} & \multicolumn{4}{|c|}{ Data input } & \multirow{3}{*}{$\begin{array}{l}\begin{array}{l}\text { Data output } \\
\text { (Reflection } \\
\text { amplitude } \\
\text { technique) }\end{array} \\
\begin{array}{l}\text { Simulated } \\
\text { dielectrics } \\
\text { (relative per- } \\
\text { mittivity) }\end{array}\end{array}$} \\
\hline & \multicolumn{2}{|c|}{ Design data } & \multicolumn{2}{|c|}{$\begin{array}{l}\text { Literature data } \\
\text { (Saarenketo 2006) }\end{array}$} & \\
\hline \multirow[t]{2}{*}{ Layer } & Thic & kness & $\begin{array}{l}\text { Relative } \\
\text { permit- } \\
\text { tivity }\end{array}$ & $\begin{array}{l}\text { Conduc- } \\
\text { tivity }\end{array}$ & \\
\hline & $\mathrm{cm}$ & inches & - & $\mathrm{S} / \mathrm{m}$ & - \\
\hline $\mathrm{AC}$ & 6 & 2.36 & 7.0 & $10^{-5}$ & 7.0 \\
\hline AC Base & 8 & 3.15 & 6.0 & $10^{-2}$ & 8.0 \\
\hline $\begin{array}{l}\text { Granular } \\
\text { Base }\end{array}$ & 20 & 7.87 & 3.0 & $10^{-1}$ & 12.8 \\
\hline Subgrade & 20 & 7.87 & 3.5 & $10^{-1}$ & - \\
\hline
\end{tabular}

\subsection{Actual dielectrics - coring data -}

The signals collected within a real-life flexible pavement are here processed by following two main approaches.

In the first one, the thicknesses of the differing layers $h_{n}$ are measured by coring, and the two-way travel time technique is used for the estimation of the permittivity of the layers. Thereby, the effective velocity of propagation $v$ within each layer can be assessed by Equation (2). Equation (1) is subsequently used for computing the actual dielectrics of the layers. Notwithstanding the intrusiveness of this approach, it is worth to emphasize how it is still considered as one of the most reliable methods for the assessment of the pavement layers dielectrics, since it relies on the measurement of the actual thicknesses. In addition, because of the better performance of the high-frequency antennas for the investigation of the dielectric properties of the surface layers (Loizos and Plati $2007 \mathrm{~b}$ ), both the $1 \mathrm{GHz}$ and the $2 \mathrm{GHz}$ data were here used for computing the layer dielectrics.

In the second approach, the amplitudes of the pulses reflected at the interfaces of the layers are related to the reference amplitude $A_{m}$ reflected by a metal sheet. The permittivity values of the layers are therefore computed for the $1 \mathrm{GHz}$ antenna using Equations (3) - (5). Table 3 lists the dielectrics found for the $\mathrm{AC}$, the $\mathrm{AC}$ base and the granular base layers using the above two approaches.

Table 3. Dielectrics of the pavement layers by the application of the two-way travel time and the reflection amplitude technique to the real-life GPR data.

\begin{tabular}{llll}
\hline $\begin{array}{l}\text { Processing } \\
\text { step II } \\
(1 \mathrm{GHz} ; 2 \mathrm{GHz})\end{array}$ & $\begin{array}{l}\text { Two-way travel time tech- } \\
\text { nique }\end{array}$ & $\begin{array}{l}\text { Reflection } \\
\text { amplitude } \\
\text { technique }\end{array}$ \\
\hline \multirow{3}{*}{ Layer } & $\begin{array}{l}\text { Relative } \\
\text { permittivity } \\
(1 \mathrm{GHZ})\end{array}$ & $\begin{array}{l}\text { Relative } \\
\text { permittivity } \\
(2 \mathrm{GHZ})\end{array}$ & $\begin{array}{l}\text { Relative } \\
\text { permittivity } \\
(1 \mathrm{GHZ})\end{array}$ \\
\cline { 2 - 4 } & - & - & - \\
\hline AC & 9.3 & 10.1 & 4.2 \\
\hline AC Base & 15.7 & 12.4 & 3.9 \\
\hline Granular Base & 5.1 & 5.1 & 3.8 \\
\hline
\end{tabular}




\subsection{Synthetic dielectrics - optimized data-}

The accuracy of the reflection amplitude method for the $1 \mathrm{GHz}$ antenna frequency is here analyzed by running an optimized FDTD simulation in the gprMax simulator. The data input for the simulation are extracted from the previous steps. In more detail, the geometric information (i.e., the thicknesses of the layers) are taken by the coring data, whereas the values of the permittivity and the conductivity are taken as the two-way travel time dielectrics by coring data (step II - two-way travel time technique) and the literature data from Saarenketo (2006), respectively. The $1 \mathrm{GHz}$ actual signal collected in real-life environment and the synthetic optimized signal are depicted in Figure 3. Table 4 lists the dielectrics computed for the layers using the reflection amplitude technique upon the simulated signal.

The bar graph in Figure 4 shows an overview of the dielectrics across the three main steps of processing performed in this study. Excluding the trend of the dataset of dielectrics for the simulation run at the step I, the application of the reflection amplitude technique provides values of the relative dielectric permittivity that progressively decrease from the surface up to the deepest layer.

Considering the dielectrics computed by the twoway travel time technique at the step II (both the 1 $\mathrm{GHz}$ and $2 \mathrm{GHz}$ data) as ground-truth reference data, it can be seen how the permittivity of the AC base layer holds the maximum value among the three

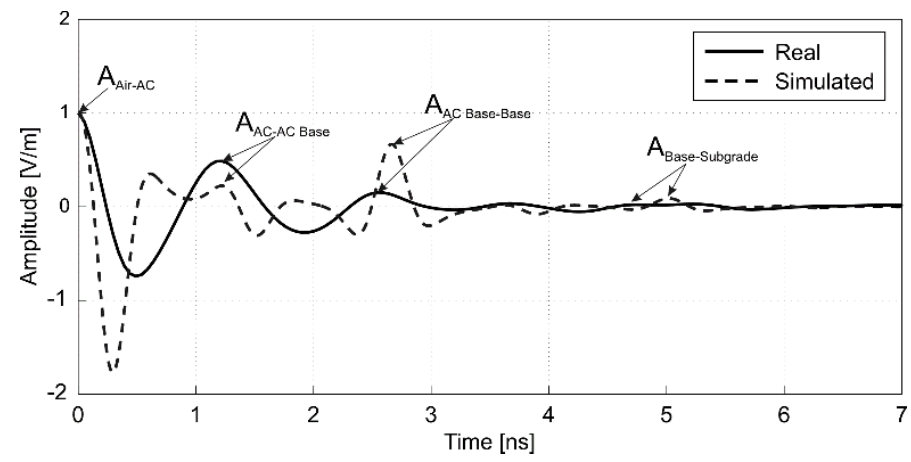

Figure 3. Comparison between the $1 \mathrm{GHz}$ actual signal (solid line) collected in the real-life environment and the $1 \mathrm{GHz}$ optimized synthetic signal (dashed line).

Table 4. Synthetic dielectrics of the pavement layers by the application of the reflection amplitude technique to the FDTD optimized signal.

\begin{tabular}{ll}
\hline $\begin{array}{l}\text { Processing } \\
\text { step III } \\
(1 \mathrm{GHz})\end{array}$ & $\begin{array}{l}\text { Reflection amplitude } \\
\text { technique }\end{array}$ \\
\hline & $\begin{array}{l}\text { Simulated } \\
\text { dielectrics } \\
\text { (relative permittivity) }\end{array}$ \\
\cline { 2 - 2 } Layer & - \\
\hline AC & 9.3 \\
\hline AC Base & 8.2 \\
\hline Granular Base & 5.2 \\
\hline
\end{tabular}

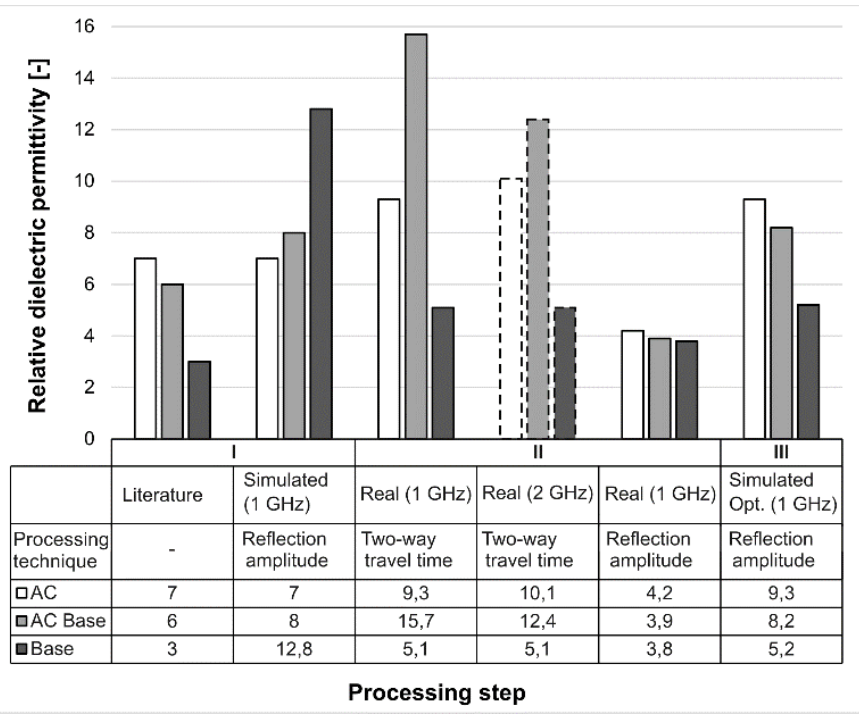

Figure 4. Relative dielectric permittivity values for the AC, AC base and base layers at the three steps of processing.

layers of the investigated pavement. This may be likely due to the intrusion of water within the AC base layer that decreases the velocity of propagation of the EM wave in the medium. Such a value decreases from $15.7(1 \mathrm{GHz})$ to $12.5(2 \mathrm{GHz})$ for higher frequencies of investigation. This non-monotonic trend of dielectrics between the three consecutive layers is not confirmed by the application of the reflection amplitude technique to both the $1 \mathrm{GHz}$ real-life and the simulated (optimized) signals.

As regards the real values of the dielectrics, the use of the reflection amplitude technique turned out to be not effective either when applied to the simulated 1 $\mathrm{GHz}$ signal (step I - design and literature data input) and the real-life $1 \mathrm{GHz}$ signal (step II). On the contrary, the use of the FDTD simulation seems to raise the accuracy of the reflection amplitude method. By comparison with the $1 \mathrm{GHz}$ real-life dataset of dielectrics (step II - two-way travel time technique), residuals of $0 \%,-47.8 \%$ and $1.9 \%$ are found for the $\mathrm{AC}$, AC base and base layers, respectively. Such residuals equal $-7.9 \%,-33.9 \%$ and $1.9 \%$ for the same layers sequence in the case of the $2 \mathrm{GHz}$ antenna (step II two-way travel time technique).

\section{CONCLUSIONS}

In this paper, the GPR technique has been used on a flexible pavement structure for the assessment of the pavement layers dielectrics. Two air-coupled GPR systems, with antennas operating at $1 \mathrm{GHz}$ and $2 \mathrm{GHz}$ central frequencies, have been utilized for investigation purposes. The combined use of design (i.e., thicknesses and construction materials) and literature data (i.e., dielectrics of the layers as per the construction material) in the simulation environment (step Isimulated signal) turned out to provide a trend of dielectrics (i.e., increasing trend) that was opposite with respect to the theoretical expectations (i.e., decreasing 
trend of the permittivity from the surface layer up to the base layers).

A combination of i) the FDTD technique for the GPR signal simulation and ii) the GPR reflection amplitude technique, has been performed for the estimation of the dielectrics of the pavement layers (step III - reflection amplitude technique). Such an approach has proved to increase the accuracy of the permittivity estimates with respect to the application of the reflection amplitude technique to the real-life signal (step II - reflection amplitude technique). This may be particularly effective when large differences between the dielectrics of the layers are found (likely related to moisture ingress within one or more layers) using the traditional two-way travel time technique and the calibration of the wave velocity of propagation by coring (step II - two-way travel time technique).

\section{ACKNOWLEDGEMENTS}

The authors would like to thank Mr Spartaco Cera, from Roma Tre University, for the support in the field work. Special thanks to IDS Georadar for supplying part of the radar equipment. This work has also benefitted from the activities carried out within the EUfunded COST Action TU1208 "Civil Engineering Applications of Ground Penetrating Radar."

\section{REFERENCES}

Al-Qadi, I.L., \& Lahouar, S. Use of GPR for thickness measurement and quality control of flexible pavements (2004) Asphalt Paving Technology: Ass. of Asphalt Paving Technologists-Proc. of the Technical Sessions, 73: 501-528.

Al-Qadi, I.L., \& Lahouar, S. Measuring layer thicknesses with GPR - Theory to practice (2005) Construction and Building Materials, 19 (10): 763-772.

Al-Qadi, I.L., Lahouar, S., Jiang, K. \& Freeman, T. E. "Effects on asphalt aging on hot-mix asphalt dielectric constants," in Proc. 83th Ann. Meeting of the Transp. Res. Board, Washington, D.C., Jan. 11-15, 2004, CD compendium.

ASTM D4748-10, Standard Test Method for Determining the Thickness of Bound Pavement Layers Using Short-Pulse Radar, ASTM International, West Conshohocken, PA 2010, http://dx.doi.org/10.1520/D4748-10 (www.astm.org).

ASTM D6087_08, Standard Test Method for Evaluating Asphalt-Covered Concrete Bridge Decks Using Ground Penetrating Radar, ASTM International, West Conshohocken, PA 2008, http://dx.doi.org/10.1520/D6087-08 /www. astm.org〉.

Bastard, C.L., Baltazart, V., Wang, Y., \& Saillard, J. Thin-pavement thickness estimation using GPR with high-resolution and superresolution methods (2007) IEEE Transactions on Geoscience and Remote Sensing, 45 (8): 2511-2519.

Benedetto, A., Tosti, F., Pajewski, L., D'Amico, F., \& Kusayanagi, W. FDTD simulation of the GPR signal for effective inspection of pavement damages (2014) Proc. of the 15th Int. Conf. on Ground Penetrating Radar, GPR 2014, art. no. 6970477, pp. 513-518.

Dobson M.C., Ulaby F.T., Hallikainen M.T. \& El-Rayes M.A. 1985. Microwave dielectric behavior of wet soil. Part II. Dielectric mixing models. IEEE Transactions on Geoscience and Remote Sensing 23: 35-46.
Fauchard, C., Côte, Ph., Le Brusq, E., Guillanton, E., Dauvignac, J.Y., \& Pichot, Ch. Step-frequency radar applied on thin road layers (2001) Journal of Applied Geophysics, 47 (3-4), pp. 317-325.

Giannopoulos, A. "Modelling ground penetrating radar by GprMax," Construction and Building Materials, vol. 19(10), pp. 755-762, 2005.

Giannopoulos, A., \& Diamanti, N. A numerical investigation into the accuracy of determining dielectric properties and thicknesses of pavement layers using reflection amplitude GPR data (2004); Proc. of the 10th Int. Conf. Ground Penetrating Radar, GPR 2004, 2, pp. 655-658.

Kunz, K.S., \& Luebbers, R.J. (1993) "The Finite Difference Time Domain Method for Electromagnetics", CRC Press.

Livneh M., \& Siddiqui M.H. Assessment of radar technology for determining the thickness of pavement layers. Proc. of the 7th Int. Conf. on Asphalt Pavements, vol. 2, Nottingham, UK, 1992, p. 228-44

Loizos, A., \& Plati, C. Accuracy of pavement thicknesses estimation using different ground penetrating radar analysis approaches (2007a) NDT and E International, 40 (2): 147-157.

Loizos, A., \& Plati, C. Accuracy of ground penetrating radar horn-antenna technique for sensing pavement subsurface (2007b) IEEE Sensors Journal, 7 (5): 842-850.

Maser, K.R. Condition assessment of transportation infrastructure using ground-penetrating radar (1996) Journal of Infrastructure Systems, 2 (2): 94-101.

Maser, K.R. \& Scullion, T. Automated Detection of Pavement Layer Thicknesses and Subsurface Moisture Using GPR. Transportation Research Board Paper, Jan. 1991.

Saarenketo, T. (2006), Electrical Properties of Road Materials and Subgrade Soils and the Use of Ground Penetrating Radar in Traffic Infrastructure Surveys, Oulu University Press, Oulu, Finland.

Roth K., Schulin R., Fluhler H. \& Attinger W. 1990. Calibration of time domain reflectometry for water content measurement using composite dielectric approach. Water Resources Research 26: 2267-2273.

Scullion, T. \& Saarenketo, T. "Integrating ground penetrating radar and falling weight deflectometer technologies in pavement evaluation," in ASTM STP 1375, Nondestructive Testing of Pavements and Backcalculation of Moduli, S. D. Tayabji and E. O. Lukanen, Eds. West Conshohocken, PA: ASTM, 2000, pp. 23-37.

Shangguan, P., \& Al-Qadi, I.L. Calibration of FDTD simulation of GPR signal for asphalt pavement compaction monitoring (2014) IEEE Transactions on Geoscience and Remote Sensing, 53 (3), art. no. 6880840: 1538-1548.

Spagnolini, U., \& Rampa, V. Multitarget detection/tracking for monostatic ground penetrating radar: Application to pavement profiling (1999) IEEE Transactions on Geoscience and Remote Sensing, 37 (1 II), pp. 383-394.

Taflove, A., (1995) "Computational Electrodynamics: The Finite-Difference Time-Domain Method", Artech House.

Tosti, F., Benedetto, A., Calvi, A. Efficient air-launched groundpenetrating radar inspections in a large-scale road network (2014); Proc. of the 3rd Int. Conf. on Transportation Infrastructure, ICTI 2014, pp. 703-709.

Willet, D.A., Mahboub, K.C., \& Rister, B., “Accuracy of ground penetrating radar for pavement-layer thickness analysis," J. Transp. Eng. ASCE, vol. 132, no. 1, pp. 96-103, 2006.

Yee, K. S. "Numerical Solution of Initial Boundary Value Problems Involving Maxwell's Equations in Isotropic Media", IEEE Transactions on Antennas and Propagation, Vol. 14, pp. 302-307, 1966.

Zhao, S., Shangguan, P., \& Al-Qadi, I.L. Application of regularized deconvolution technique for predicting pavement thin layer thicknesses from ground penetrating radar data (2015) NDT and E International, 73, art. no. 1674, pp. 1-7. 